\title{
Identification of a subtle $t(16 ; 19)(p 13.3 ; p 13.3)$ in an infant with multiple congenital abnormalities using a 12-colour multiplex FISH telomere assay, M-TEL
}

\author{
Jill Brown ${ }^{1}$, Sharon W Horsley ${ }^{1}$, Christine Jung $^{2}$, Kaan Saracoglu ${ }^{3}$, Bart Janssen ${ }^{2}$, \\ Michaela Brough ${ }^{2}$, Markus Daschner ${ }^{4}$, Bernd Beedgen ${ }^{4}$, Guido Kerkhoffs ${ }^{4}$, Roland Eils ${ }^{3}$, \\ Peter C Harris ${ }^{5}$, Anna Jauch ${ }^{2}$ and Lyndal Kearney ${ }^{1}$
}

\begin{abstract}
${ }^{1}$ M RC M olecular Haematology Unit, Institute of Molecular Medicine, Oxford, UK; ${ }^{2}$ Institute for Human Genetics, University of Heidelberg, Germany; ${ }^{3}$ D epartment of Intelligent Bioinformatics Systems, German Cancer Research Center, Heidel berg, Germany; ${ }^{4}$ Pediatric D epartment, University of Heidel berg, Germany; ${ }^{5}$ Department of Nephrology, Mayo Clinic, Rochester, MN, USA
\end{abstract}

There is increasing evidence that cytogenetically invisible chromosome rearrangements are an important cause of genetic disease. Clues to the chromosomal location of these rearrangements may be provided by a specific clinical diagnosis, which can then be investigated by targeted FISH or molecular studies. However, the phenotypic features of some microdeletion syndromes are difficult to recognise, particularly in infants. In addition, the presence of other chromosome aneuploidy may mask the typical clinical features. In the present study, the presence of tubers on cranial magnetic resonance imaging (MRI) of a 5-week-old infant prompted an investigation, by FISH, with probes from the tuberous sclerosis gene, TSC2. This and further FISH deletion mapping studies revealed a submicroscopic deletion encompassing the entire TSC2 gene and the adjacent PKD1 gene on one chromosome 16, confirming a del(16)(p13.3). Because of the large number of abnormal phenotypic features in this infant, we performed a 12-colour FISH assay (M-TEL) to screen for subtelomeric rearrangements involving the del(16p). The M-TEL assay revealed a cryptic der(16)t(16;19)(p13.3;p13.3). Further FISH with 19p and 19q subtelomeric probes demonstrated that this was derived from a balanced maternal $t(16 ; 19)(p 13.3 ; p 13.3)$. Importantly, 24-colour painting by multiplex FISH (M-FISH) failed to detect the translocation in either the infant or his mother. Based on our FISH mapping studies, we estimate the size of the trisomic region from 19p13.3 to be approximately $2 \mathrm{Mb}$, and the region of monosomy for $16 \mathrm{p} 13.3$ as $2.25 \mathrm{Mb}$. This case adds to the growing literature which indicates that many apparent chromosomal deletions are unbalanced translocations. The M-TEL assay provides a sensitive alternative to M-FISH for the detection of these subtle telomeric rearrangements. European Journal of Human Genetics (2000) 8, 903-910.

Keywords: TSC2, PKD1 deletions; multiplex FISH; subtelomeric probes

\section{Introduction}

Even with high resolution banding, conventional (G-banded) cytogenetic analysis can only detect chromosome rearrangements $>3 \mathrm{Mb}$. However, there is increasing

Correspondence: Dr Lyndal Kearney, MRC Molecular Haematology Unit, Institute of Molecular Medicine, Oxford, OX3 9DS, UK.

Tel: +44 1865 222389; Fax: +44 1865 222500;

E-mail: Ikearney@hammer.imm.ox.ac.uk

Received 31 March 2000; revised 29 June 2000; accepted 3 July 2000 evidence that apparently normal karyotypes may harbour subtle chromosome rearrangements, particularly involving the G-band negative telomeric chromosome regions. Using a FISH-based screening assay and a set of chromosome-specific subtelomeric probes (Multiprobe telomere assay), one study reported the identification of subtle telomeric chromosome rearrangements in $7.4 \%$ of children with moderate to severe mental retardation. ${ }^{1}$ In all cases, the G-banded karyotype had been reported as normal. Other studies have used the 
24-colour karyotyping techniques, multiplex-FISH (M-FISH) and spectral karyotyping (SKY), to reveal hidden chromosome rearrangements. ${ }^{2,3}$ However, the resolution of both $\mathrm{M}-\mathrm{FISH}$ and SKY is limited, particularly for telomeric rearrangements. ${ }^{2,4}$ The Multiprobe telomere assay consists essentially of 23 dual-colour hybridisations carried out on a single microscope slide. ${ }^{5}$ However, to ensure 5-10 high quality metaphases on each of the 23 chromosome 'squares', this assay requires a high mitotic index and is therefore not applicable in all clinical situations. We report here the application of an alternative multicolour telomere screening assay, M-TEL, which allows the full survey of all telomeric regions in only two hybridisations. This provides a significant advantage over the Multiprobe assay in cases where the mitotic index is low, such as in many leukaemic bone marrow samples, or where the clinical material is limited. In the present study, the M-TEL assay was used to investigate an apparently normal karyotype in a child with multiple features suggestive of a chromosomal disorder. These included dysmorphism, evidence of tuberous sclerosis, craniosyntosis, colobomas of the iris, hypospadias and cryptorchidism, inguinal hernias and $\alpha$ thalassaemia. The initial finding of tubers on cranial magnetic resonance imaging (MRI) prompted an investigation using FISH with probes for the TSC 2 gene. This identified a del(16)(p13.3) and led us to investigate the possibility of the involvement of a cryptic translocation involving this chromosome.

\section{Clinical report}

The proband is a male patient, the second child of a healthy, 27-year-old mother and the first child of a healthy, 31-yearold father. The 4-year-old half-brother is also healthy. The parents are unrelated. The pregnancy with the proband was un remarkable until the 36th week, when oligohydramnios was noted by ultrasound and the foetal cardiotocograph was abnormal. The foetus was in the breech position and a Caesarean section was performed. The child was vital, Apgar $7^{1}, 10^{10}$. Measurements at birth were as follows: birthlength $42 \mathrm{~cm}$ (3rd centile), birthweight $1550 \mathrm{~g}$ ( $<<3 \mathrm{rd}$ centile), head circumference $30.5 \mathrm{~cm}$ (10th centile). Muscular hypotonia was noted and the sucking reflex was poor. Respiratory problems led to oesophageal $\mathrm{pH}$ measurement, which revealed gastro-oesophageal reflux, probably causing recurrent aspiration. The problem resolved with inclined positioning and physiotherapy.

A striking dolichocephalic head configuration (Figure1) was evaluated by radiography, which showed a synostosis of the posterior part of the sagittal suture. A cranial ultrasound showed a normal configuration of the ventricles and no pathological findings. Convulsions occurred at the age of 5 weeks and a cranial MRI revealed bilateral subcortical and subependymal areas of el evated signal intensity suggestive of tubers. Treatment with phenobarbitone was in effective, but vigabatrin reduced seizure frequency.
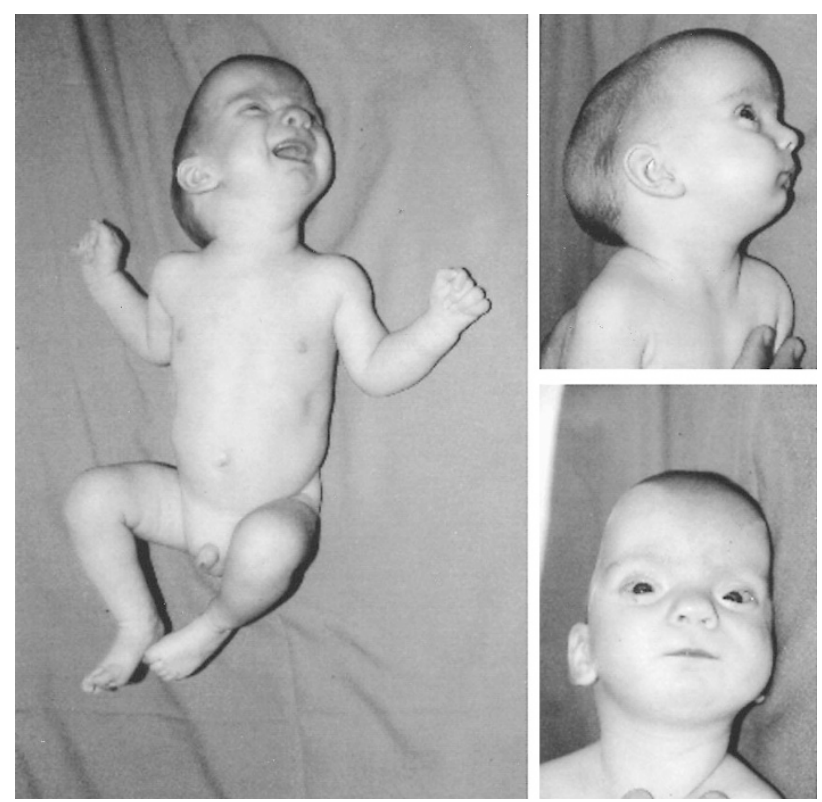

Figure 1 The proband at age 4 months, showing opisthotonus, dolichocephaly and facial dysmorphism.

Bilateral nasal colobomas were present, but the remainder of the ophthalmological investigation was normal. Aural examination and brainstem-evoked response audiometry was normal. At 3 weeks, mildly elevated creatinine levels $(0.6 \mathrm{mg} / \mathrm{dl})$ prompted renal ultrasonography that revealed hyperechogenic kidneys with several small, subcortical renal cysts. Echocardiography at the age of 2 weeks gave normal results and excluded rhabdomyomas.

Haematological investigation showed a hypochromic, microcytic anaemia. Haemoglobin electrophoresis at the age of 7 weeks showed $\mathrm{HbA}_{2}$ : $1.1 \%$ (normal range $<3 \%$ ), $\mathrm{HbF}$ $27.2 \%$ (within the age-dependent normal range), Hb Bart's $4 \%$, indicating reduced synthesis of $\alpha$ globin. This is compatible with $\alpha$ thalassaemia.

The clinical examination at the age of 4 months showed a boy of $58 \mathrm{~cm}$ length (25th centile), $3960 \mathrm{~g}$ weight ( $3 \mathrm{rd}$ centile), and $39 \mathrm{~cm}$ head circumference (10th centile). He presented with muscular hypertonia and opisthotonus. He had bilateral inguinal hernias, glandular hypospadias and bilateral cryptorchidism. Additional dysmorphic signs included telecanthus, mild exorbitism, short palpebral fissures, broad nasal tip, small mouth with thin lips and small chin. The ears were low set and posteriorly rotated, but not dysplastic. The nipples were inverted. The skin was dry and pale with a leaflet hypopigmentation of $0.5 \mathrm{~cm}$ length on the left calf. The hands and feet were normal.

Physical examination of the parents revealed no major abnormalities. The mother showed striae of the buttocks and thighs, one café-au-lait spot of maximum $3 \mathrm{~cm}$ diameter at the left flank, one of maximum $2 \mathrm{~cm}$ diameter on the top of the right shoulder and a naevus flammeus of the nuchal 
region. There were no cutaneous features of tuberous sclerosis in either parent.

\section{Materials and methods Cytogenetic analysis}

Peripheral blood metaphase chromosomes were prepared from the proband and both parents by standard procedures. GTG banding was performed at the $450-500$ band level using standard protocols. Twelve metaphases each from the proband and both of the parents were analysed.

\section{FISH}

FISH was carried out using standard protocols. ${ }^{6,7}$ The probes used for deletion mapping of 16p13.3 were (listed from telomere to centromere): cos GG4 (containing the $\alpha$ globin upstream regulatory element HS-40), cos GG1 (containing the entire $\alpha$ globin gene cluster), cos 313F2 and plasmid CW 23 (from the 3 ' and 5 ' ends of the TSC 2 gene respectively), $\cos \mathrm{JH} 2 \mathrm{~A}$ and $\cos 2 \mathrm{H} 2$ (both from the duplicated region of $16 \mathrm{p} 13.3$ which includes part of the PKD1 gene), cosmids CW40I and 218C (centromeric to PKD1), and two cosmid probes for the Rubinstein-Taybi gene, RT100 and RT203 ${ }^{8}$ (see Figure 2a). A 19p PAC containing the E2A gene, PAC $1116 F 22$ was provided by Mariano Rocchi, Resources for Molecular Cytogenetics, Bari (http://bioserver.uniba.it/fish/Cytogenetics) to localise the 19p13.3 breakpoint.

\section{M-TEL assay}

A second generation set of PAC, BAC, P1 and cosmid clones was used to identify all chromosome ends (except the acrocentric $p$ arms) (Table1). The majority of these are confirmed as within $500 \mathrm{~kb}$ of their respective chromosome end. $^{9}$ The M-TEL assay is described in detail elsewhere ${ }^{10}$. Briefly, probes were combinatorially labelled by nick translation using digoxigenin (detected with FITC), Cy3, biotin (detected with Cy3.5) and estradiol (detected with Cy5.5), with both the $p$ and $q$ arm probes for each chromosome labelled with the same fluorochrome combination (Table2). Two separate hybridisations were performed, identifying 12 (M-TEL1) and 11 (MTEL-2) chromosome pairs, respectively. Images were collected using a Sensys CCD camera (KAF1400 chip, Photometrics, Tucscon, USA) mounted on an Olympus AX 70 epifluorescence microscope (Olympus Optical Co.,

(a)

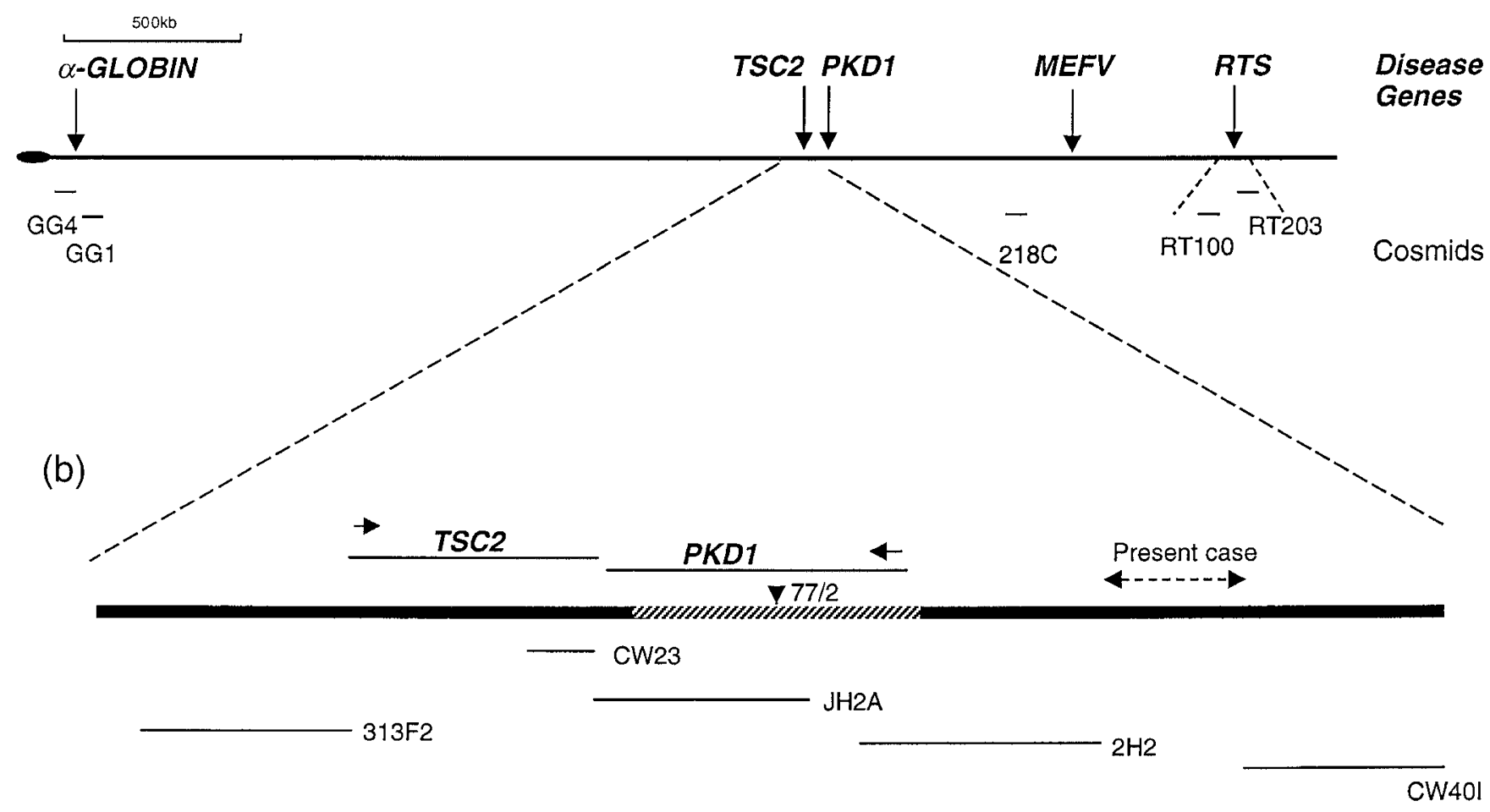

Figure 2 Identification of the 16p13.3 breakpoint. a Long-range map of the terminal region of 16p, showing the disease genes (TSC2, tuberous sclerosis; PKD1, polycystic kidney disease; M EFV, familial Mediterranean fever; RTS, Rubinstein Taybi syndrome), and the cosmids used to map the extent of 16 p13.3 deletion. b A detailed map of the region surrounding the TSC2 and PKD 1 genes and the probes used to refine the breakpoint in this case. The area of $16 \mathrm{p} 13.3$ duplicated in $16 \mathrm{p} 13.1$ is also indicated (hatched). The translocation breakpoint previously found in family $77^{23}$ is shown by an arrowhead. The breakpoint region in the present case lies between cosmids $2 \mathrm{H} 2$ and $\mathrm{CW} 40 \mathrm{O}$ (indicated by a dashed line). 
Table 2 Combinatorial labelling scheme for the M-TEL

Table 1 Subtelomeric probes used in the M-TEL assay

\begin{tabular}{|c|c|c|c|}
\hline Telomere & Clone name & Clone type & Reference \\
\hline $1 p$ & 14e10 & PAC & 9 \\
\hline 19 & $160 \mathrm{H} 23$ & PAC & 9 \\
\hline $2 p$ & $892 \mathrm{G} 20$ & PAC & 9 \\
\hline $2 q^{a}$ & 1011017 & PAC & 9 \\
\hline $3 p$ & $1186 \mathrm{~B} 18$ & PAC & 9 \\
\hline $3 q$ & 196F4 & PAC & 9 \\
\hline $4 p$ & $36 \mathrm{P} 21$ & PAC & 9 \\
\hline $4 q$ & $963 K 6$ & PAC & 9 \\
\hline $5 p^{a}$ & $114 J 18$ & BAC & 9 \\
\hline $5 q$ & $240 \mathrm{G} 13$ & PAC & 9 \\
\hline $6 \mathrm{p}$ & $62 \mid 11$ & PAC & 9 \\
\hline $6 q$ & $57 \mathrm{H} 24$ & PAC & 9 \\
\hline $7 p$ & 164D18 & PAC & 27 \\
\hline $7 q$ & $3 K 23$ & PAC & 9 \\
\hline $8 p^{a}$ & $790 \mid 10$ & PAC & 9 \\
\hline $8 q$ & 489D14 & PAC & 9 \\
\hline $9 p$ & 51L14 & BAC & 9 \\
\hline $10 p$ & 305F4 & PAC & 9 \\
\hline $10 \mathrm{q}$ & $137 \mathrm{e} 24$ & PAC & 9 \\
\hline $11 p^{a}$ & $908 \mathrm{H} 22$ & PAC & 9 \\
\hline 119 & $1064 \mathrm{e} 20$ & PAC & 9 \\
\hline $12 p^{a}$ & 496A11 & PAC & 9 \\
\hline $12 q$ & $221 \mathrm{~K} 18$ & PAC & 9 \\
\hline $13 q$ & $85 A 10$ & P1 & 27 \\
\hline $14 q$ & $820 M 16$ & PAC & 27 \\
\hline $15 q$ & $154 \mathrm{P} 1$ & PAC & 9 \\
\hline $16 p$ & 119L16 & PAC & 27 \\
\hline $16 q$ & $240 \mathrm{G} 10$ & PAC & 27 \\
\hline $17 p$ & 2111b1 & cosmid & 5 \\
\hline $17 q^{a}$ & B37c1 & cosmid & 5 \\
\hline $18 p$ & $52 \mathrm{M} 11$ & P1 & 27 \\
\hline $18 q$ & $964 \mathrm{M} 9$ & PAC & 27 \\
\hline $19 p$ & $546 C 11$ & PAC & 9 \\
\hline $19 q$ & $129 c 9$ & PAC & 9 \\
\hline $20 p$ & $1061 / 1$ & PAC & 9 \\
\hline $20 q$ & $81 F 12$ & PAC & 9 \\
\hline $21 q$ & $63 \mathrm{H} 24$ & PAC & 9 \\
\hline $22 q$ & $99 K 24$ & PAC & 9 \\
\hline XpYp & $98 c 4$ & PAC & 9 \\
\hline
\end{tabular}

aWeak cross hybridisation with one or two other telomeres. This was usually restricted to one chromosome homologue and only present in a proportion of cells. However, the $9 \mathrm{q}$ and Xq PACs crosshybridised strongly with several other telomeres, and were therefore excluded from the M-TEL hybridisation mixtures. For these chromosome ends it was necessary to use the first generation cosmids applied in a separate hybridisation.

London, UK), with an eight position filter turret containing filters specific for DAPI, FITC, Cy3, Cy3.5, Cy5.5 (Chroma Technology, Brattlebro, USA). The camera was controlled by MacProbe v 4.0 software (PSI, Chester, UK). Analysis of the combinatorially labelled probes was performed using two software analysis programmes: (i) a modified version of MacProbe v 4.1, which allowed karyotyping of the inverted DAPI image and sequential viewing of the individual fluorochrome channels in karyotyped format, and (ii) a modified version of the spectral imaging approach originally devised for $\mathrm{M}-\mathrm{FISH} .{ }^{11}$ This conceptually new analysis approach (goldFISH ${ }^{\mathrm{TM}}$ ) performs classification of telomeres according to their fluorochrome combination after identification of telomeres by anisotropic non-linear diffusion filtering. ${ }^{12}$ assay

\begin{tabular}{|c|c|c|c|c|}
\hline Telomeres & Dig-FITC & Су3 & Вio-Суз.5 & Est-Cy5.5 \\
\hline \multicolumn{5}{|l|}{ M-TEL1 } \\
\hline $1 p$ and $q$ & & $x$ & & \\
\hline $3 p$ and $q$ & $x$ & & $x$ & \\
\hline $5 p$ and $q$ & & $x$ & & $x$ \\
\hline $7 p$ and $q$ & & $x$ & $x$ & \\
\hline $9 p$ & $x$ & & & $x$ \\
\hline $11 p$ and $q$ & $x$ & $x$ & & \\
\hline $13 q$ & $x$ & $x$ & $x$ & \\
\hline $15 q$ & & & $x$ & $x$ \\
\hline $17 p$ and $q$ & $x$ & & & \\
\hline $19 p$ and $q$ & & & & $x$ \\
\hline $21 q$ & $x$ & & $x$ & $x$ \\
\hline XpYp & & & $x$ & \\
\hline \multicolumn{5}{|l|}{ M-TEL2 } \\
\hline $2 \mathrm{p}$ and $\mathrm{q}$ & $x$ & & & \\
\hline $4 p$ and $q$ & $x$ & & $x$ & \\
\hline $6 p$ and $q$ & & $x$ & & \\
\hline $8 p$ and $q$ & $x$ & & & $x$ \\
\hline $10 p$ and $q$ & & $x$ & $x$ & \\
\hline $12 p$ and $q$ & $x$ & $x$ & & \\
\hline $14 p$ & & & $x$ & \\
\hline $16 p$ and $q$ & & $x$ & & $x$ \\
\hline $18 p$ and $q$ & & & & $\mathrm{X}$ \\
\hline $20 p$ and $q$ & & & $x$ & $x$ \\
\hline $22 q$ & $x$ & & $x$ & $\mathrm{X}$ \\
\hline
\end{tabular}

Dig, digoxigenin; Bio, biotin; Est, estradiol. The two sets of probes required for a full telomere survey are termed M-TEL1 and M-TEL2, respectively.

\section{4-colour whole chromosome painting and M-FISH analysis}

M-FISH was performed using a set of combinatorially labelled whole chromosome paints. ${ }^{11}$ The hybridisation conditions and image analysis were as previously described. ${ }^{11}$ Images were collected using a motorised epifluorescence mi croscope with an eight-position filter wheel (Leica DMRXA-RF8) and a Sensys CCD camera (Photometrics, Tucson, USA). M-FISH analysis was performed using Leica MCK software (Leica Microsystems Imaging Solutions). Both the microscope and camera were controlled by Leica Q-FISH software (Leica Microsystems Imaging solutions). ${ }^{11}$

\section{Results}

\section{Cytogenetic analysis}

G-banded cytogenetic analysis of peripheral blood metaphases showed the proband's karyotype to be $46, X Y$. The karyotypes of both parents were also normal. A partial karyotype of chromosomes 16 and 19 from the mother and the proband is shown in Figure 3.

\section{FISH}

The 16p13.3 probes GG4, GG1, 313F2, CW23, JH2A and 2H2 all showed fluorescent signal on one chromosome 16 homologue only. However, CW40I, 218C, RT100 and RT203 all showed fluorescent signal on both chromosomes 16, placing the 16 p13.3 breakpoint between $2 \mathrm{H} 2$ and CW40I (Figure2b). 


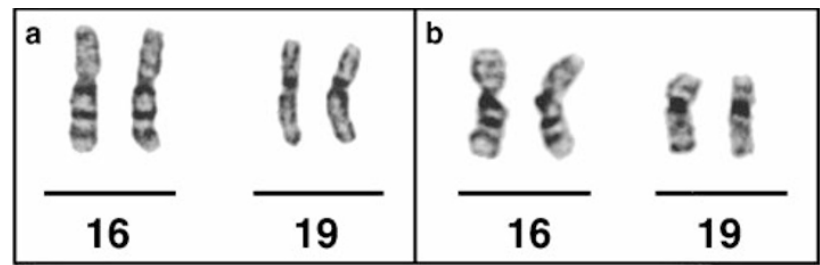

Figure 3 A representative G-banded partial karyotype of chromosomes 16 and 19 from the proband a and her mother b No abnormality is visible in either the balanced $\mathbf{b}$ or unbalanced form of the translocation $\mathbf{a}$.

This confirmed a deletion encompassing the entire $\alpha$ globin gene cluster, the TSC2 gene and the adjacent PKD1 gene. Although this FISH analysis indicates that the entire PKD 1 gene is deleted, detailed molecular analysis is needed to identify the exact breakpoint. Based on physical mapping studies of 16p13.3 (R Daniels, personal communication 2000), we estimate the size of the deleted region to be $2.25 \mathrm{Mb}$.

\section{M-TEL}

The M-TEL1 hybridisation showed an additional chromosome19 signal on the abnormal chromosome16 (Figure4). One further dual-colour FISH experiment with $19 p$ and $19 q$ subtelomeric probes showed that this was 19p. The M-TEL2 hybridisation confirmed deletion of the $16 p$ subtelomeric probe. FISH carried out on the parental chromosomes using $16 p$ and 19p subtelomeric probes confirmed that this unbalanced translocation was derived from a maternal $t(16 ; 19)$ (Figure5). Therefore, the revised karyotype of the proband is: 46,XY,der(16)t(16;19)(p13.3;p13.3)mat.

\section{M-FISH}

24-colour whole chromosome painting and M-FISH analysis failed to detect the translocation in metaphases from either the proband or the mother.

\section{Discussion}

There is increasing evidence that many apparent telomeric deletions are unbalanced translocations, revealed only by molecular or molecular cytogenetic techniques. ${ }^{4,13-15}$ There is also a number of cases which have a phenotype suggestive of a genetic abnormality, but which do not fit any known syndrome. The 24-colour karyotyping techniques M-FISH and SKY provide one approach to whole genome screening for chromosome abnormalities, but are limited in resolution. In the present study, M-FISH (performed retrospectively, once the rearrangement had been identified) was unable to detect a rearrangement involving $2.25 \mathrm{Mb}$ of $16 \mathrm{p} 13.3$. Other studies have estimated the resolution of $\mathrm{M}-\mathrm{FISH}$ to be between $300 \mathrm{~kb}$ and $2.6 \mathrm{Mb}$, based on the visibility of the Xp, but not the $\mathrm{Xq}$ pseudoautosomal regions. ${ }^{2}$ However, this resolution depends on a number of other factors including the band level of the chromosomes and the fluorochrome composition of the respective chromosome paints, so that absolute levels cannot be set. Comparative genomic hybridisation (CGH) provides another approach to whole genome screening, but can only detect deletions of $>10 \mathrm{Mb}^{16}$ and is particularly unsuitable for the detection of telomeric rearrangements. The application of $\mathrm{CGH}$ to an array of mapped DNA sequences immobilised on a glass slide (DNA microarray) promises to increase this resolution to detect deletions of $40-80 \mathrm{~kb}^{17,18}$ At present however, DNA microarray technology is not available in most clinical diagnostic situations. The M-TEL assay provides a sensitive, specific approach which will detect deletions, as well as balanced and unbalanced transl ocations involving the telomeric regions. However, one limitation of the assay in its present format is that it will not discriminate pericentric inversions, as both the $p$ and $q$ arms are detected in the same colour. Intrachromosomal rearrangements such as this require a multicolour banding approach such as cross-species colour banding ${ }^{19}$ or multicolour bar-coding. ${ }^{2}$

The translocation identified in this case results in monosomy for 16p13.3 and trisomy for 19p13.3. These are both G-band negative, GC-rich isochores which fit the criteria of T-bands (a subset of R-bands containing the highest density of $\mathrm{CpG}$ islands). ${ }^{20}$ Rearrangements involving these two regions might therefore be expected to produce a significant effect. The telomeric band $16 \mathrm{p} 13.3$ contains a number of genes which, when present in only one copy, cause disease. These include the $\alpha$ globin gene cluster, tuberous sclerosis (TSC2), autosomal dominant polycystic kidney disease (PKD1), and Rubinstein Taybi syndrome (RTS) genes. The 16p deletion in the present case includes the entire PKD 1 and TSC2 genes, which lie immediately adjacent. Disruption of both TSC 2 and PKD 1 genes is associated with a severe renal phenotype presenting in infancy. ${ }^{21,22}$ These patients have bilateral polycystic renal disease and often grossly enlarged kidneys. Hence, the PKD is more severe than that associated with mutation to just PKD 1, which normally results in adult onset disease; suggesting a synergistic role for the PKD 1 and TSC 2 products in cyst development. ${ }^{22}$ Follow-up examination of the patient at 9 months showed normal size kidneys, with bilateral small cysts, and that his serum creatinine had fallen to within normal levels. Although the renal cystic disease here is less severe than in the majority of cases with TSC2/PKD 1 deletions, other such patients with cystic disease only detected by ultrasound have been described. ${ }^{22}$ Indeed, the appearance of multiple cysts in such a young infant is an indication of severe disease and similar to that reported in the index case in family 77, in which TSC2 is deleted and PKD 1 disrupted due to a translocation..$^{23}$ In family 77 the balanced translocation carriers also had polycystic kidneys, because PKD1 was disrupted, but in the present case renal ultrasound did not show any evidence of polycystic kidneys in the mother. These results indicate, consistent with the FISH data, that the breakpoint lies 5' to the PKD 1 gene and 


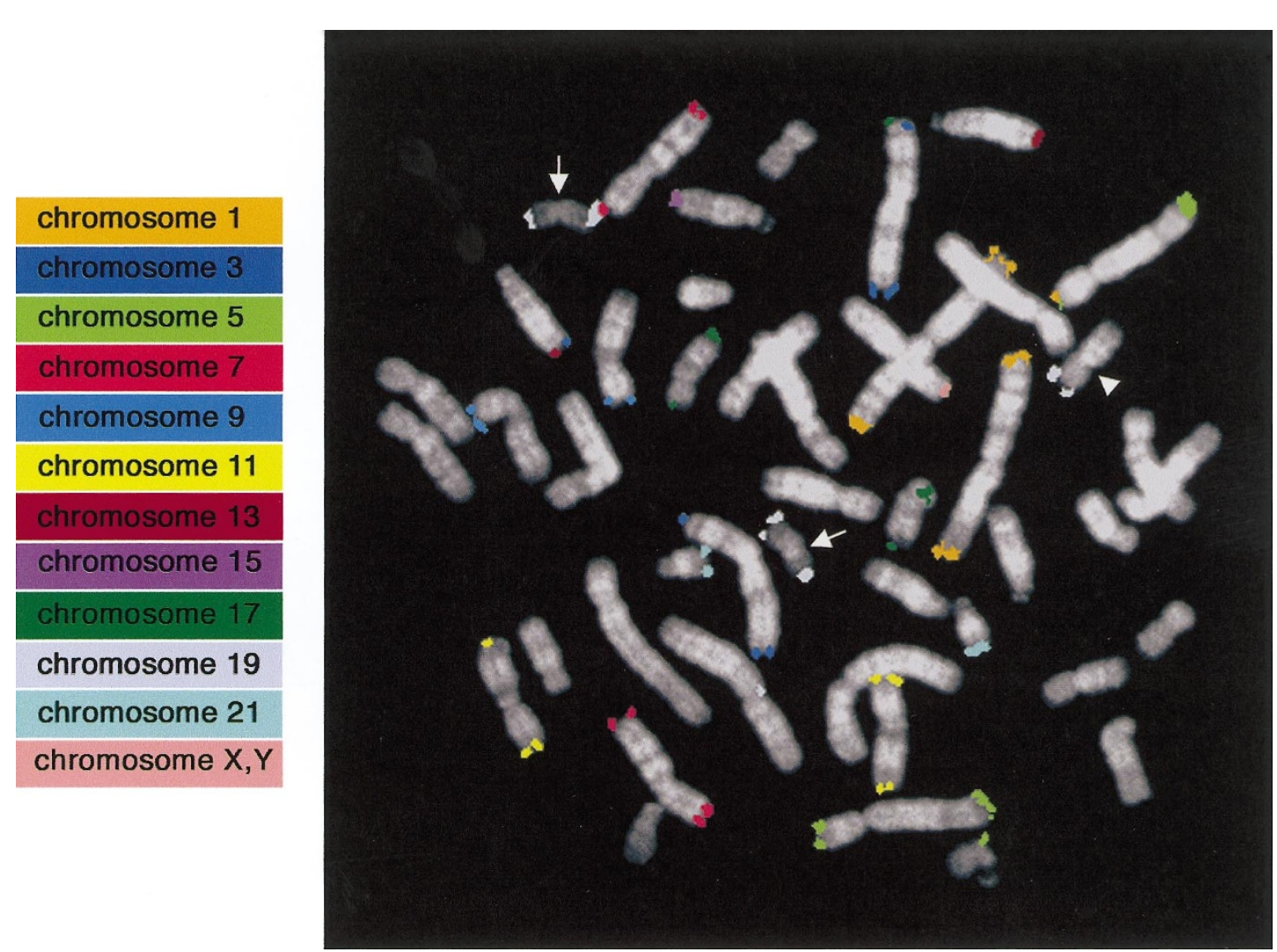

Figure 4 Colour classification (goldFISH ${ }^{\mathrm{TM}}$ ) analysis of the telomeric signals in the M-TEL1 hybridisation to a metaphase from the proband. This identifies subtelomeric sequences corresponding to chromosome 19 on the der(16) (arrowhead). Arrows indicate that the two normal chromosome 19 homologues. The respective pseudocolours are given on the left. There are some apparent misclassifications, all involving only one out of a possible four chromatids on one chromosome homologue. For example, one chromosome 5 homologue has the signal on one $\mathrm{p}$ arm chromatid classified as chromosome 1 (orange). As the labelling scheme for chromosomes 1 and 5 differs only by the presence of one fluorochrome (Cy5.5) (see Table2), the absence of a Cy5.5 signal would result in the 'wrong' colour classification. Similarly, one chromatid each of chromosomes 3 and 13 has been assigned the wrong colour. However, in all of the above chromosomes, a signal for the 'missing' fluorochromes was present (although weak) when the individual fluorochrome images were viewed. A weaker signal on one chromatid probably reflects the fact that the fluorochrome images were in slightly different focal planes. This demonstrates the importance of being able to review the individual fluorochrome images to resolve such discrepancies. Note also that in all cases the misclassification involved only one out of a possible four chromatids. In establishing the M-TEL assay, we set the minimum requirement for accurate classification as three out of four possible chromatids with the correct classification. ${ }^{12}$

beyond elements required for normal PKD1 expression. Precise localisation of the breakpoint would give a clearer idea of the size of the 5' region that allows normal PKD1 expression.

The 19p13.3 breakpoint was within a PAC containing the E2A gene, which lies approximately $2 \mathrm{Mb}$ from the $19 \mathrm{p}$ telomere (http://greengenes.llnl.gov/genome-bin/loadmap?region $=\mathrm{mp}$ ). Chromosome 19 has the highest ratio (for its size) of gene-based marker assignments of any human autosome. ${ }^{24,25}$ Therefore, it is expected that trisomy for even a small portion of this chromosome would produce a significant phenotype. There are only two previous reports of trisomy 19p (reviewed in Salbert et $\mathrm{al}^{26}$ ). Both of these apparently involved a larger region of trisomy from 19p (19p13.2) and both also had concurrent monosomy for other (different) chromosome regions. The features common to both of these cases were intra-uterine growth retardation, small palpebral fissures and ear anomalies. However, it is difficult to relate these features to those of the present case.

This is yet another example of a cryptic subtelomeric rearrangement associated with multiple congenital abnormalities. In this case, the features were highly suggestive of tuberous sclerosis, which allowed a targeted FISH approach and led to the initial identification of del(16)(p13.3). Neither G-banding nor M-FISH (performed retrospectively after the translocation had been identified) was sensitive enough to detect the translocation. The M-TEL assay provides a sensitive and flexible alternative to both M-FISH and the currently 


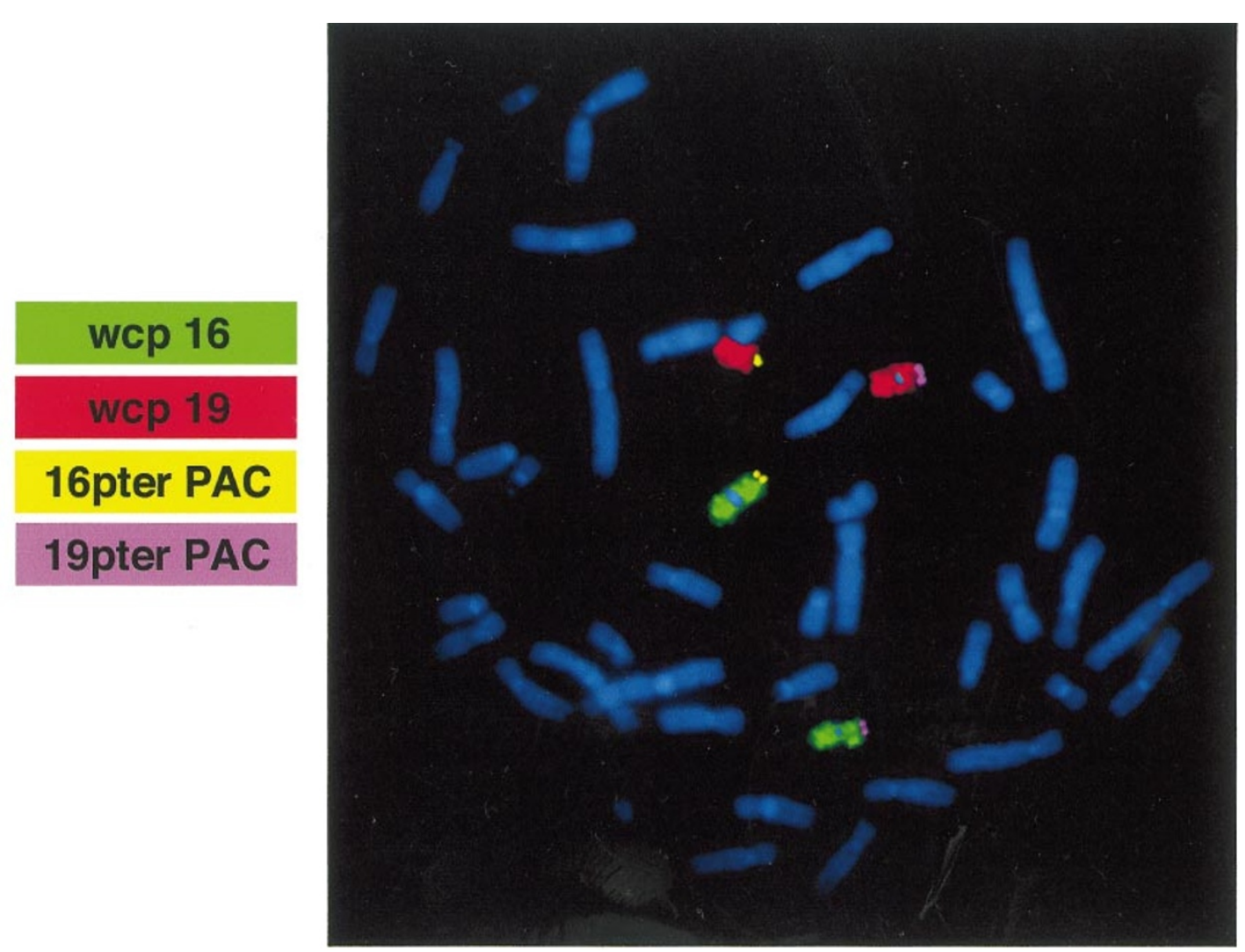

Figure 5 Four-colour FISH to demonstrate the presence of a balanced $t(16 ; 19)$ in the mother of the proband. Whole chromosome paints (wcp) for chromosomes 16 (directly labelled with Cy5, pseudo-coloured green) and 19 (labelled with estradiol and detected with Cy5.5, pseudo-coloured red), as well as subtelomeric PACs for 16pter (biotin labelled, detected with FITC, pseudo-coloured yellow) and 19pter (labelled with digoxigenin, detected with Cy3, pseudo-coloured pink) were co-hybridised.

available methods for subtelomeric probe screening. It is expected that this approach will have a wide range of applications for both clinical diagnosis and research.

\section{Acknowledgements}

We are grateful to Sam Knight, Sarah Lucas and Regina Regan (IMM, Oxford) for the second generation subtel omeric probes, D oug Higgs and Rachael Daniels (IMM, Oxford) for the $\alpha$ globin gene probes, and Mariano Rocchi (Bari) for supplying the E2A PAC. We also thank Andrew Wilkie (IMM, Oxford) for critical reading of the manuscript. This work was supported in part by the Leukaemia Research Fund, UK (JB), the Medical Research Council (LK), Deutsche Forschungsgemeinschaft grant JA-688/2-1 and Wilhelm Sander-Stifung grant 98/025.1 (AJ). R Eils acknowledges support from the German Minister for Education and Research (BMBF, BioFuture grant AZ 11880), Deutsche Forschungsgemeinschaft (Ja 395/6-2, Ei 358/1-1) and the German-Israeli foundation for research and technology (GIF G-112-207.04/97).

\section{References}

1 Knight SJL, Regan R, Horsley SW et al: Subtle chromosomal rearrangements in children with unexplained mental retardation. Lancet 1999; 354: 1676-1681.
2 Uhrig S, Schuffenhauer S, Fauth C et al: Multiplex-FISH for preand postnatal diagnostic applications. Am J Hum Genet 1999; 65: 448-462.

3 Schröck E, Veldman T, Padilla-Nash H et al: Spectral karyotyping refines cytogenetic diagnostics of constitutional chromosomal abnormalities. Hum Genet 1997; 101: 255-262.

4 Holinski-Feder E, Reyniers E, Uhrig $S$ et al: Familial mental retardation syndrome ATR-16 due to an inherited cryptic subtelomeric translocation, $\mathrm{t}(3 ; 16)(\mathrm{q} 29 ; \mathrm{p} 13.3)$. Am J Hum Genet 2000; 66: $16-25$.

5 Knight SJL, Horsley SW, Regan R et al: Development and clinical application of an innovative fluorescence in situ hybridization technique which detects submicroscopic rearrangements involving telomeres. Eur J Hum Genet 1997; 5: 1-8.

6 Lichter P, Cremer T: Chromosome analysis by non-isotopic in situ hybridization. In: Rooney DE, Czepulkowski BH (eds). Human Cytogenetics. Constitutional Analysis: A Practical Approach, vol 1, Oxford University Press: Oxford, 1992, pp 157-190.

7 Buckle VJ, Rack K: Fluorescent in situ hybridization. In: Davies KE (ed). Human Genetic Disease Analysis. IRL Press: Oxford, 1993, pp 59-82.

8 Giles RH, Petrij F, Dauwerse HG et al: Construction of a 1.2-Mb contig surrounding, and molecular analysis of, the human CREBbinding protein (CBP/CREBBP) gene on chromosome16p13.3. Genomics 1997; 42: 96-114.

9 Knight SJL, Lese CM, Kuc J et al: An optimized set of human telomere clones for studying telomere integrity and architecture. Am J Hum Genet 2000; 67: 320-332. 
10 Brown J, Saracoglu K, Uhrig S, Speicher MR, Eils R, Kearney L: Subtelomeric chromosome rearrangements are detected using 12-colour multiplex FISH assay (M-TEL). Nature Med (in press).

11 Eils R, Uhrig S, Saracoglu K et al: An optimized, fully automated system for fast and accurate identification of chromosomal rearrangements by multiplex-FISH (M-FISH). Cytogenet Cell Genet 1998; 82: 160-171.

12 Saracoglu K, Brown J, Kearney L, Uhrig S, Speicher MR, Eils R: New software concepts and probe design improve resolution and sensitivity of molecular cytogenetics by M-FISH. Cytometry (submitted).

13 Lamb J, Wilkie AOM, Harris PC et al: Detection of breakpoints in submiscroscopic chromosomal translocation, illustrating an important mechanism for genetic disease. Lancet 1989; 2: 819-824.

14 Horsley SW, Knight SJL, Nixon J et al: Del(18p) shown to be a cryptic translocation using a multiprobe FISH assay for subtelomeric chromosome rearrangements. J Med Genet 1998; 35: 722-726.

15 Praphanphoj V, Goodman BK, Thomas GH, Raymond GV: Cryptic subtelomeric translocations in the 22q13 deletion syndrome. J Med Genet 2000; 37: 58-61.

16 Bentz M, Plesch A, Stilgenbauer S, Döhner H, Lichter P: Minimal sizes of deletions detected by comparative genomic hybridization. Genes, Chromosomes Cancer 1998; 21: 172-175.

17 Solinas-Toldo S, Lampel S, Stilgenbauer $S$ et al: Matrix-based comparative genomic hybridization: biochips to screen for genomic imbalances. Genes, Chromosomes Cancer 1997; 20: 399-407.

18 Pinkel $D$, Seagraves $R$, Sudar $D$ et al: High resolution analysis of DNA copy number variation using comparative genomic hybridization to microarrays. Nat Genet 1998; 20: 207-211.
19 Müller S, Rocchi M, Ferguson-Smith MA, Wienberg J: Toward a multicolor chromosome bar code for the entire human karyotype by fluorescence in situ hybridization. Hum Genet 1997; 100: 271-278.

20 Craig JM, Bickmore WA: The distribution of CpG islands in mammalian chromosomes. Nat Genet 1994; 7: 376-382.

21 Brook-Carter PT, Peral B, Ward CJ et al: Deletion of the TSC2 and PKD1 genes associated with severe infantile polycystic kidney disease - a contiguous gene syndrome. Nat Genet 1994; 8: 328-332.

22 Sampson JR, Maheshwar MM, Aspinwall R et al: Renal cystic disease in tuberous sclerosis: role of the polycystic kidney diseasel gene. Am J Hum Genet 1997; 61: 843-851.

23 European Polycystic Kidney Disease Consortium: The polycystic kidney disease 1 gene encodes a $14 \mathrm{~kb}$ transcript and lies within a duplicated region on chromosome16. Cell 1994; 77: 881-894.

24 Cross SH, Lee M, Clark VH, Craig JM, Bird AP, Bickmore WA: The chromosomal distribution of $\mathrm{CpG}$ islands in the mouse: evidence for genome scrambling in the rodent lineage. Genomics 1997; 40: 454-461.

25 Deloukas P, Schuler GD, Gyapay G et al: A physical map of 30,000 human genes. Science 1998; 282: 744-746.

26 Salbert BA, Solomon M, Spence JE, Jackson-Cook C, Brown J, Bodurtha J: Partial trisomy 19p: case report and natural history. Clin Genet 1992; 41: 143-146.

27 National Institutes of Health and Institute of Molecular Medicine Collaboration: A complete set of human telomeric probes and their clinical application. Nat Genet 1996; 13: 86-89. 